\title{
Philosophiques
}

\section{Laberge, P., La théologie kantienne précritique, Éditions de l'Université d'Ottawa, 1973, 192 p.}

\section{Olivier Reboul}

Volume 1, numéro 2, octobre 1974

URI : https://id.erudit.org/iderudit/203015ar

DOI : https://doi.org/10.7202/203015ar

Aller au sommaire du numéro

Éditeur(s)

Société de philosophie du Québec

ISSN

0316-2923 (imprimé)

1492-1391 (numérique)

Découvrir la revue

Citer cet article

Reboul, O. (1974). Laberge, P., La théologie kantienne précritique, Éditions de l'Université d'Ottawa, 1973, 192 p. Philosophiques, 1(2), 83-93.

https://doi.org/10.7202/203015ar d'utilisation que vous pouvez consulter en ligne.

https://apropos.erudit.org/fr/usagers/politique-dutilisation/ 


\section{ÉTUDES CRITIQUES}

LABERGE, P., La théologie kantienne précritique, Éditions de l'Université d'Ottawa, 1973, 192 p.

\section{par Olivier REBOUL}

KANT AVANT KANT : ainsi pourrait s'intituler l'ouvrage de Pierre Laberge, consacré à l'évolution de la métaphysique de Kant, de sa première oeuvre à la période critique. Sans doute l'auteur limite-t-il son champ de recherche à la théologie naturelle, mais l'étude de Dieu et de ses preuves l'amène à traiter de la plupart des problèmes qui intéressent le métaphysicien : ontologie, cosmologie (et cosmogonie !), théologie, théodicée.

Les trois premiers chapitres étudient respectivement $L$ 'bistoire générale de la nature et la théorie du ciel, de 1755, la Nova dilucidatio, thèse latine soutenue la même année, et l'Unique fondement possible d'une démonstration de l'existence de Dieu (Beweisgrund), de 1763. Etude minutieuse et précieuse, car le lecteur français ne dispose d'aucune traduction de la première de ces oeuvres, et pour la seconde de la seule version de Tissot de 1852 , d'ailleurs fort contestable. Un quatrième chapitre aborde la question, pour nous décisive, du rapport entre la théologie précritique et de la théologie morale de la période critique.

L'auteur avertit modestement le «lecteur pressé » (p. 10) qu'il peut se dispenser de lire les trois premiers chapitres, analytiques, et se contenter de la synthèse du dernier. Je crois que, même pressé, le lecteur perdrait beaucoup à sauter le premier chapitre, 
consacré à la première oeuvre de Kant, et qui nous révèle un visage du philosophe aussi insolite qu'éclairant pour la suite.

L'bistoire générale développe un seul argument en faveur de l'existence de Dieu: la preuve par l'ordre de l'univers, entendu comme l'harmonie entre le mécanisme naturel et l'avantage des être raisonnables. Kant expose et critique la preuve par la finalité, si populaire au XVIIIe siècle: si certains phénomènes naturels apparaissent comme providentiels pour l'homme, la nature dans son ensemble comporte trop d'imperfections pour qu'on puisse en conclure à la perfection de son auteur. Le mécanisme de Newton pourrait fournir à la preuve une base beaucoup plus sûre, parce qu'universelle; mais Newton lui-même n'a pu mener à terme son hypothèse explicative: l'origine du mouvement tangentiel, notamment, reste mystérieuse; Kant n'hésite pas à corriger Newton en créant «une cosmogonie mécaniste» (p. 19) qui déduit l'ordre du monde des propriétés fondamentales de la matière et prouve a posteriori l'existence d'un Dieu omnisuffisant comme l'auteur de cet ordre absolu. Fondé sur le modèle atomiste, la cosmogonie de Kant rappelle celle d'Epicure; elle se veut comme elle pleinement mécaniste ; comment donc peut-elle conduire à Dieu? C'est que le mécanisme est la négation même du hasard, qu'Epicure plaçait à l'origine de tout; le mécanisme, qui rend toute la nature intelligible, ne peut être que le produit d'une intelligence : "Il y a un Dieu justement parce que la nature même dans le chaos ne peut se comporter que légalement et avec ordre », conclut Kant (cf. p. 46).

P. Laberge ne cache pas les faiblesses de cette preuve téléologique remise à jour : non seulement l'ordre du monde pourrait bien ne prouver qu'un «artiste immanent > (p. 41), mais encore cet ordre même, Kant est obligé de le supposer en grande partie pour lui donner l'universalité requise par la preuve.

Et quand je dis supposer ... Kant, en nous expliquant comment le monde a pu naître et se développer, se livre à un véritable exercice de science fiction, affirmant même que les planètes doivent être habitées, et qu'en vertu des lois physiques constitutives du système solaire le corps de ces habitants, ainsi que leur esprit qui en dépend, est d'autant plus parfait que leur planète domi- 
ciliaire est plus éloignée du soleil ; les habitants de Neptune sont donc infiniment plus grossiers que les terriens, ceux de Saturne infiniment plus spirituels; entre ces brutes et ces anges, l'homme se trouve dans un entre-deux redoutable, privé de l'inconscience de la brute comme de la félicité de l'ange. On appréciera le savoureux résumé (p. 26 ss) des arguments de Kant en faveur de son hypothèse.

Celle-ci a pourtant un prolongement dans la philosophie pratique de Kant: l'affirmation que l'homme est l'être à la fois raisonnable et fini. $P$. Laberge souligne lui-même les affirmations de l'Histoire générale qui annoncent la philosophie critique: le principe ramenant toute légalité naturelle à un entendement législateur; le principe selon lequel le monde est engagé, de par ses propres lois, dans un progrès à l'infini (sur ce point Laberge s'oppose à ceux qui voient dans ce livre de Kant une théorie du retour éternel); enfin le principe, que l'on retrouvera à la base de toute la théologie pratique, posant que la justification du monde est la considération des êtres raisonnables. L'auteur montre bien comment dans l'Histoire générale ces principes portent à faux; la métaphysique qui était alors celle de Kant n'était pas propice à leur accueil. Ainsi Kant affirmait que la considération de l'être raisonnable est la vraie justification de l'univers - « La fin dernière de l'existence du monde » dira la Critique du juge. ment - mais, en bon leibnizien, il déclarait dans cette même oeuvre que la justification de l'univers est la gloire de Dieu, et que les misères de l'homme "n'avaient rien de scandaleux, puisqu'elles s'inscrivaient à leur place dans un ensemble qui valait par sa variété » (p. 117). L'homme fin pour Dieu ou l'homme moyen de Dieu? Kant ne peut encore décider entre ces deux théodicées, apparemment contraires.

Ce premier chapitre, si riche et si clair, me laisse insatisfait sur un point d'histoire. L'auteur affirme, avec Kant lui-même d'ailleurs (cf. Critique de la raison pure, P.U.F., p. 362-363) «qu'Epicure représente le tenant des antithèses de l'Antinomie » (p. 29 ; cf. p. 148). Epicure était pourtant le tenant d'un monde fini (du moins dans l'espace), de l'indivisibilité absolue des atomes, de la liberté comme cause première! La seule antithèse 
dont on puisse le créditer est la quatrième, celle qui nie l'existence de Dieu, comme être nécessaire cause du monde.

\section{II}

Les chapitres II et III étudient respectivement la Nova Dilucidatio et le Beweisgrund, toujours en fonction des preuves de l'existence de Dieu. Malgré leurs différences, que l'auteur analyse de façon précise, ces deux oeuvres retiennent l'une et l'autre quatre arguments: la preuve cartésienne, la preuve ontothéologique, la preuve wolffienne et la preuve physico-théologique. Kant, dans les deux oeuvres, rejette la première et la troisième, admet partiellement la quatrième tout en considérant la seconde - qui est d'ailleurs son oeuvre originale - comme seule démonstrative.

L'argument cartésien n'est autre que la preuve ontologique de la Critique de la raison pure. Il est intéressant de retracer l'évolution de Kant à son égard. Dans la Nova Dilucidatio il l'expose ainsi : la notion d'un être qui contient l'omnitudo realitatis (la totalité du réel) prouve l'existence de cet être, car l'existence elle-même est une realitas. Kant admet la logique de l'argument ; si Dieu existait, son essence impliquerait son existence; mais le conditionnel indique bien qu'il ne s'agit là que d'une existence représentée: penser Dieu, c'est penser qu'il existe, mais je ne sors pas de ma pensée! Dans le Beweisgrund, la critique est plus radicale, et déjà celle de la Dialectique transcendantale: on ne peut même plus conclure de la pensée de Dieu à celle de son existence, car l'existence n'est pas de l'ordre de la pensée; Kant dénonce l'ambiguitté du langage, qui nous laisse croire que «Dieu est » et «Dieu est bon » mettent l'être sur le même plan, qu'il soit prédicatif ou existentiel. L'existence n'est pas un prédicat qui s'ajouterait aux perfections de Dieu, car l'existence n'est pas un prédicat du tout; elle est «la position absolue d'une essence déjà riche de toutes ses déterminations » (p. 84).

$\mathrm{P}$. Laberge montre bien que la véritable opposition entre Kant et Descartes se joue à propos de l'idée de causa sui. Si Dieu existe nécessairement, pour Descartes, c'est qu'il est de son essence d'être cause de soi-même. Kant répond — un peu légèrement, à mon gré - que cette idée de cause de soi est absurde. 
L'argument wolffien, a contingentia mundi, n'est autre que la preuve cosmologique de la Critique de la raison pure. Si la Nova Dilucidatio la rejette avec hauteur, le Beweisgrund en donne un examen approfondi dont les conclusions diffèrent de celles de la Critique. Kant admet en effet les deux premières étapes de la preuve: 1) si quelque chose existe, il existe aussi quelque chose qui ne dépend de rien, car on ne peut admettre une régression à l'infini dans la série des causes. 2) Cette chose incausée existe de façon absolument nécessaire, sinon elle aurait une cause ; la Critique nous interdira de passer ainsi de la causalité phénoménale à une cause nouménale; ici Kant remarque simplement qu'on pose à tort la nécessité absolue de ce qui n'est nécessaire qu'en fonction du monde. 3) En tout cas, comme dans la Critique, il rejette la troisième étape de la preuve, qui identifie cette existence absolument nécessaire à l'omnitudo realitatis, identification qui suppose admis l'argument cartésien; sinon, comment savoir que la cause première est aussi l'être parfait? On remarque déjà le génie critique de Kant, qui décèle dans la preuve l'argument implicite qui seul la rend probante, et qui, réfuté, l'entraîne dans sa propre chute.

L'argument physico-théologique n'apparaît pas comme tel dans la Nova Dilucidatio; on trouve à la place une preuve par la communauté des substances qui doit avoir son principe hors de celles-ci, donc en Dieu. À titre d'exemple, de cette communauté, Kant donne la localisation dans l'espace et l'attraction newtonienne. D'où la remarque fort pertinente de l'auteur au sujet du premier exemple : Kant explique, en 1755, par un schème dans l'entendement divin «l'être en relation réciproque des substances dans l'espace que la Critique de la raison pure rendra tributaire d'une synthèse transcendantale de l'imagination aux ordres du Ich denke » (p. 77, cf. p. 120). De l'Etre nécessaire au Je transcendantal, de Dieu à l'homme, telle est l'évolution de la pensée kantienne.

L'argument physico-théologique, le troisième dans la Critique de la raison pure, n'apparaît vraiment que dans le Beweis. grund, qui reprend le problème du sens du monde là où l'Histoire générale l'avait laissé. À nouveau, Kant critique la conception populaire et celle des finalistes: pourquoi ne pas dire que Dieu 
nous a donné un nez pour chausser nos lunettes! Le fondement de la preuve n'est pas dans la finalité, toujours partielle et ambiguë, mais dans la légalité universelle qui régit le monde. C'est l'unité de l'univers qui prouve, ou plutôt suggère, la perfection de son auteur; c'est le mécanisme de l'horloge et non son utilité qui témoigne pour l'horloger. La faiblesse de la preuve, Kant le remarque déjà, est qu'elle permet de conclure à un Dieu architecte, non au Dieu créateur. Ici encore on peut remarquer l'évolution de Kant: cette unité du monde, qui dans le Beweisgrund renvoie à un entendement divin, deviendra dans la Critique l'unité a priori de l'expérience, qui renvoie au Je transcendantal.

Reste le problème de la théodicée. Dans le Beweisgrund, Kant se refuse à sacrifier à la considération de l'être raisonnable la toute-puissance et l'omni-suffisance divines dont témoigne le mécanisme universel. D'où une «théodicée impitoyable» (p. 145) qui va jusqu'à prétendre que si la disparition du monde découlait nécessairement de ses propres lois (si l'entropie devait totalement triompher dirions-nous), il ne faudrait pas demander à Dieu, comme Newton, de le remettre en marche! Car un résultat déterminé par les lois de la nature ne peut être que bon (cf. p. 116 et 119). Si les fins du Créateur servent l'être raisonnable, celui-ci ne peut raisonnablement savoir comment. Dans de fortes pages, $P$. Laberge montre que, pour le Kant précritique comme pour le Kant de la Critique du jugement, la finalité ne se donne qu'à celui qui renonce à la chercher, que le sens du monde est un sens certain mais caché : «Il faut perdre la finalité pour la gagner $\gg$ (p. 173 ; cf. 124 ss).

Bref, la célèbre critique kantienne des trois preuves de l'existence de Dieu est déjà en place dans le Beweisgrund de 1763. Le problème est que ce livre avance une autre preuve, la preuve ontothéologique, qu'il considère justement comme l'unique fondement d'une démonstration de Dieu. Or cette unique démonstration, la Critique de la raison pure ne la mentionne pas, pas même pour la critiquer. Etrange problème, en vérité. 
III

C'est précisément à résoudre ce problème que $\mathrm{P}$. Laberge consacre tout son effort, un effort qui constitue sans doute le tonos de son livre et lui donne son unité profonde.

L'argument ontothéologique, qu'on trouve dans la Nova Dilucidatio comme dans le Beweisgrund est proprement kantien. Le problème de Leibniz et de Wolff était de montrer la conve nance de tous les possibles; Kant pose une question plus radicale comment se fait-il qu'il existe des possibles? Si l'idée du triangle était contradictoire avec celle de l'angle droit, il serait certes impossible de se représenter un triangle rectangle; mais la représentation serait également impossible si triangle et angle droit n'existaient pas. L'existence des possibles est le Reale, ou Realitas, bien distincts pour Kant de la Wirklichkeit, ou existence empirique et contingente: le réel, c'est le pensable. Comment donc se fait-il qu'il existe du pensable? Le second temps de la preuve montrera que tout possible - ou réel, ou pensable - dérive par limitation d'un être absolument nécessaire, qui constitue la totalité du réel et qui est Dieu. Démarche qui repose sur le principe que la négation n'est pas première, et que toutes les réalités, limitées parce qu'elles impliquent une négation, impliquent la réalité illimitée dans laquelle elle se découpent. Dieu est donc l'omnitudo realitatis, la totalité du réel, et toute existence limitée ne se comprend qu'à partir de lui.

P. Laberge montre fort bien comment le modèle de l'omnitudo realitatis va devenir, dans la Critique, celui de l'espace: chaque morceau d'espace n'est possible que par l'espace, totalité unique dont chaque espace n'est qu'une partie. Ici encore, on passe de l'ontologique au transcendantal.

Ce qui intéresse surtout l'auteur est de savoir quel Dieu prouve l'argument ontothéologique. Ce Dieu, omnitudo realitatis, n'est-il pas le Dieu de Spinoza? Car, s'il comprend tous les possibles, il comprend donc l'étendue, l'impénétrabilité, et:. L'innovation du Beweisgrund sera justement d'éluder cette conséquence et de reprendre la preuve pour montrer que le Dieu auquel elle aboutit n'est pas l'omnitudo realitatis, mais l'Ens realissimum, n'est pas le fond où se découpent tous les êtres mais 
le fondement de tous les êtres. P. Laberge discute longuement la démarche du Beweisgrund, qui consiste à établir que l'étendue, par les oppositions réelles qu'elle comporte, ne peut être Dieu, mais dérivée de Dieu. Démarche artificielle, nous dit-il, et dont la Critique sanctionnera l'échec (cf. p. 102-103).

Ce que montre surtout P. Laberge, c'est l'enjeu du débat. Si le Dieu auquel aboutit le seul argument admis par Kant est le Dieu de Spinoza, toute la question est de savoir comment Kant a finalement évité d'être spinoziste. Car ce qui ressort du présent ouvrage, c'est que la pensée «précritique», malgré sa problématique et son lexique leibniziens, est en réalité plus proche de Spinoza que de Leibniz. Spinoziste, la conclusion «naturelle 》 de l'unique argument en faveur d'un Dieu comme totalité du réel; spinoziste, l'affirmation que Dieu est la source nécessaire non seulement des existences, mais des essences, alors que le Dieu de Leibniz n'est en réalité qu'un démiurge en face des possibles; spinoziste, le primat du mécanisme sur la finalité; spinoziste enfin, le rejet de tout anthropomorphisme, la solution du problème du mal (et de la liberté humaine) par « une théodicée impitoyable » (p. 145).

Le rapprochement est encore plus évident quand on envisage comment Spinoza lui-même rejetait la conséquence à laquelle semblait le conduire son panthéisme; l'identification de Dieu à la nature et au monde matériel. Deus sive natura: oui, mais nature naturante, non naturée; Dieu n'est pas pour Spinoza la somme des réalités, mais l'activité infinie qui les produit comme ses modes; et s'il a l'étendue comme attribut, c'est que l'étendue divine est dépourvue de toute divisibilité et de toute inertie, de même que la pensée divine est sans imagination et sans passions ; Dieu est tout, mais tout ce qui est négatif n'est rien en Dieu, c'est-à-dire rien en soi. Il est significatif, en tout cas, comme le note $\mathrm{P}$. Laberge, que la Critique de la raison pratique présentera le spinozisme «comme la seule solution de rechange à l'idéalisme transcendantal $\gg(\mathrm{p} .175)^{\mathrm{I}}$.

1. Cf. Critique de la raison pratique, P.U.F., p. 107-108, ou Vrin, 114-115. 
Comment Kant a-t-il pu écarter la solution spinoziste pour admettre un Dieu spirituel et créateur? Telle est la question du dernier chapitre. Mais là, dans le souci louable de ne pas dépasser les limites que lui assignait son titre, l'auteur reste un peu en retrait, me semble-t-il, de la ligne où son lecteur l'attendait. Ses réflexions sur les deux premières Critiques sont plus timides que celles qui précèdent.

La thèse de Laberge est que l'argument ontothéologique (l'unique preuve) n'a pas disparu dans la Critique de la raison pure: on le retrouve dans la section célèbre, et combien obscure, sur «l'Idéal transcendantal». Obscure, car l'Idéal (l'idée de Dieu) est à la fois omnitudo realitatis et ens realissimum. L'idéalisme transcendantal est la véritable réfutation de l'argument ontothéologique, puisqu'il rend impossible l'attribution d'un objet à ce qui n'est qu'un idéal de la raison; la force de l'argument reste intacte, mais elle se situe au niveau de l'expérience: "Rien n'est pour nous un objet, s'il ne suppose l'ensemble de toute la réalité empirique comme condition de sa possibilité » ( $\mathrm{Cr}$. de la $r$. pure, p. 420). Par une illusion naturelle, nous projetons l'unité de l'expérience dans le monde intelligible pour l'hypostasier en unité divine. Maintenant, la Critique de la raison pratique, qui prouve la nécessité de croire en Dieu comme postulat de la morale, ne va-t-elle pas rendre l'existence à l'Idéal transcendantal et en assu mer l'ambiguïté?

Non, répond P. Laberge, car l'idéal transcendantal, comme omnitudo realitatis, «est naturellement spinoziste» (p. 180) et le Dieu que requiert la morale est un Dieu personnel, spirituel et créateur.

On ne peut que souscrire à cette thèse. Mais faut-il ajouter que Kant passe du Dieu panthéiste au Dieu personnel par un virage illégitime, une «volte-face» (p. 168, cf. 181)? Qu'il n'échappe qu'arbitrairement aux conclusions qu'impliquait son système, et qui affirmaient le Dieu des philosophes au détriment du Dieu des croyants (cf. p. 181)?

Quant à moi, je ne poserais pas l'alternative en ces termes. Le Dieu des philosophes n'est pas nécessairement celui de Spinoza. 
Descartes, en affirmant un Dieu absolument libre et cause de soi, échappe au panthéisme de l'omnitudo realitatis et montre que les prédicats de la matière sont en Dieu non pas «formellement», mais «éminemment»; ce qui permet à Dieu de créer le monde et l'homme comme son vis-à-vis, sans rien aliéner de sa toutepuissance. D'autre part, le Dieu des croyants n'est pas le dispensateur automatique de châtiments et de récompenses que suggèrent certains textes de la Critique de la raison pratique. $\mathrm{P}$. Laberge (cf. p. 171) aurait pu citer d'autres passages de Kant, en particulier dans ses dernières oeuvres, qui attribuent à Dieu non seulement la justice mais la grâce et l'amour.

Je pense surtout que l'option de Kant pour la foi en un Dieu personnel et créateur n'est pas arbitraire. $P$. Laberge, après avoir si bien montré comment les attributs de Dieu dans la pensée précritique deviennent dans la critique les attributs de l'homme comme Je transcendantal et sujet moral, aurait pu montrer qu'une telle intériorisation approfondit non seulement l'idée de l'homme mais l'idée de Dieu. L'idéalisme transcendantal nous interdit d'hypostasier l'omnitudo realitatis, qui ne vaut que pour l'expérience. Il montre également que le seul objet d'un savoir métaphysique est la liberté humaine, démontrée par les lois de la raison pratique (cf. Critique du jugement, $\mathrm{n}^{\circ}$ 91); or la liberté exclut, non pas arbitrairement mais logiquement, le panthéisme, car le panthéisme la rendrait impossible. Le Kant précritique cherchait la preuve de Dieu dans l'admirable mécanisme du ciel étoilé; le Kant critique la trouve dans une certitude beaucoup plus radicale, celle de la loi morale en nous. Et le Dieu qui sert de répondant à la loi morale et à la liberté humaine ne peut être un tout mais une personne.

Bref, ce dernier chapitre nous laisse un peu sur notre faim. Mais, si l'on admet avec Nietzsche que le propre d'un maître est de donner une faim, ma critique peut être finalement un éloge. Après avoir magistralement retracé l'itinéraire de la théologie kantienne jusqu'à son point d'aboutissement, P. Laberge laisse son lecteur avec le souci et le désir d'approfondir lui-même cet aboutissement.

Une oeuvre d'érudition dominée, d'érudition au service d'une pensée vivante, qui s'interroge sans cesse en gardant sans cesse le 
cap qu'elle s'est fixé. Une oeuvre indispensable, tant pour ceux qui s'intéressent à Kant que pour ceux qui cherchent à philosopher pher sur Dieu.

Université de Montréal 